\title{
Certain results on Ricci solitons in $\alpha$-Kenmotsu manifolds
}

\author{
Rajesh Kumar ${ }^{1}$, Ashwamedh Mourya ${ }^{2}$ \\ IDepartment of Mathematics, Pachhunga University College, Aizawl 796oor, India \\ ${ }^{2}$ Department of Mathematics, Ashoka Institute of Technology and Management, Varanasi 221007, India
}

\begin{abstract}
In this paper, we study some curvature problems of Ricci solitons in $\alpha$-Kenmotsu manifold. It is shown that a symmetric parallel second order-covariant tensor in a $\alpha$-Kenmotsu manifold is a constant multiple of the metric tensor. Using this result, it is shown that if $\left(L_{v} g+2 S\right)$ is parallel where $V$ is a given vector field, then the structure $(g, V, \lambda)$ yield a Ricci soliton. Further, by virtue of this result, Ricci solitons for $n$-dimentional $\alpha$-Kenmotsu manifolds are obtained. In the last section, we discuss Ricci soliton for 3-dimentional $\alpha$-Kenmotsu manifolds.
\end{abstract}

2010 Mathematical Subject Classification: 53C25, 53C10, 53C44

Key words: Ricci soliton, $\alpha$-Kenmotsu manifold, Einstein manifold.

\author{
Received o7 November 2017 \\ Accepted 06 January 2018 \\ *For correspondence $\triangle$ : \\ rajesh_mzu@yahoo.com \\ Contact us $\bowtie$ : \\ sciencevision@outlook.com \\ https://doi.org/10.33493/scivis.18.01.02
}

\section{Introduction}

A Ricci soliton are the natural generalization of Einstein metric and are defined on a Riemannian manifold. On the manifold $M$, a Ricci soliton is a triple ${ }^{1}(g, V, \lambda)$ with a Riemannian metric $g$, a vector field $V$ and a real scalar $\lambda$ such that

$\left(\mathcal{L}_{V} g\right)(X, Y)+2 S(X, Y)+2 \lambda g(X, Y)=0$,

for any vector fields $X, Y$ on $\chi(M)$ where $S$ is the Ricci tensor and $\mathcal{L}_{V}$ denotes the Lie derivative operator along the vector field $V$. The metric satisfying (1) are very interesting in the field of physics and are often referred as quasiEinstein.2,3 The Ricci soliton is said to be shrinking, steady and expanding according as $\lambda$ is negative, zero and positive respectively. ${ }^{4}$

Das ${ }^{5}$ studied second order parallel tensor on an almost contact metric manifold and found that on an $\alpha$-K-contact manifold ( $\alpha$ being nonzero real constant) a second order symmetric parallel tensor is a constant multiple of the associative positive definite Riemannian metric tensor. It is also proved that in an $\alpha$-Sasakian manifold there is no non-zero parallel 2-form. The study of Ricci solitons in K-contact manifolds was started by Sharma ${ }^{6}$ and in the continuation of this Ghosh, Sharma and $\mathrm{Cho}^{7}$ studied gradient Ricci soliton of a non-Sasakian $(k, \mu)$-contact manifold. Generally, in a PSasakian manifold the structure vector field $\xi$ is not killing, that is $\left(\mathcal{L}_{V} g\right) \neq 0$ but in $\mathrm{K}$-contact manifold $\xi$ is a killing vector field, that is $\left(\mathcal{L}_{V} g\right)=$ 0 . Recently, $\mathrm{De}^{8}$ have studied Ricci soliton in PSasakian, Barua and $\mathrm{De}^{9}$ have studied Ricci soliton in Riemannian manifolds. Since then several other studied Ricci soliton have been published in various contact manifolds: Eisenhart problem to Ricci soliton in $f$ Kenmotsu manifold, ${ }^{10}$ Eta-Ricci solitons on paraKenmotsu manifolds, ${ }^{11}$ on contact and Lorentzian manifolds, ${ }^{10,12,13}$ on Sasakian manifold, ${ }^{14,15} \alpha$-Sasakian manifold, ${ }^{16}$ on Kenmotsu manifold, ${ }^{17}$ etc.

Motivated by above studies, in this paper we treat Ricci soliton in $\alpha$-Kenmotsu manifolds. The paper is structured as follows. After 
introduction, section 2 is a brief review of $\alpha$ Kenmotsu manifold. Section 3, is devoted to the study of parallel symmetric second order tensor in $\alpha$-Kenmotsu manifold and Ricci soliton in $\alpha$ Kenmotsu manifolds. In this section, we obtain a relation between symmetric parallel second order covariant tensor and metric tensor in $\alpha$ Kenmotsu manifold. In the second problem of this section we studied the necessary and sufficient condition of a Ricci semi-symmetric $\alpha$ Kenmotsu manifold and $\eta$-Einstein manifold Section 4 is devoted to study Ricci soliton in 3dimensional $\alpha$-Kenmotsu manifold.

\section{$\alpha$-Kenmotsu manifold}

An $n$-dimensional real $C^{\infty}$-manifold $M$ is said to almost contact structure $(\varphi, \xi, \eta)$ if it admits a $(1,1)$ tensor field $\varphi$, a contravariant vector field $\xi$ and a 1-form $\eta$ which satisfy

$$
\eta(\xi)=1, \varphi^{2} X=-X+\eta(X) \xi \text {, }
$$

which implies

$$
\varphi(\xi)=0, \eta(\varphi X)=0,
$$

for all vector field $X, Y$ on $\chi(M)$, where $\chi(M)$ is the Lie algebra of $C^{\infty}$ vector fields on $M$. An $n$ dimensional real $C^{\infty}$-manifold $M$ equipped with almost contact structure $(\varphi, \xi, \eta)$ is called almost contact manifold ${ }^{18}$.

An almost contact manifold $M$ with metric tensor $g$ which satisfies the condition

$$
g(\varphi X, \varphi Y)=g(X, Y)-\eta(X) \eta(Y),
$$

and $g(X, \xi)=\eta(X)$,

is called almost contact metric manifold $M$ $(\varphi, \xi, \eta, g)$.

An almost contact metric manifold $M$ is said to be almost $\alpha$-Kenmotsu manifold if

$$
d \eta=0, \quad \text { and } \quad d \Phi=2 \alpha \eta \wedge \Phi,
$$

where $\Phi$ is a fundamental 2-form defined as $\Phi(X, Y)=g(\varphi X, Y)$ and $\alpha$ being a non-zero rea] constant. ${ }^{17}$ Moreover, if an almost $\alpha$-Kenmotsu manifold $M$ satisfies the following relations

$\left(\nabla_{X} \varphi\right) Y=-\alpha\{g(X, \varphi Y) \xi+\eta(Y) \varphi X\}$

and $\left(\nabla_{X} \xi\right)=\alpha\{X-\eta(X) \xi\}$,

then it is called $\alpha$-Kenmotsu manifold. ${ }^{17,18,19}$

On an $\alpha$-Kenmotsu manifold $M$, the following relations hold ${ }^{20,21,22}$

$$
\begin{aligned}
& R(X, Y) \xi=\alpha^{2}\{\eta(X) Y-\eta(Y) X\}, \\
& R(\xi, X) Y=\alpha^{2}\{\eta(Y) X-g(X, Y) \xi\}, \\
& \eta(R(X, Y) Z)=\alpha^{2}\{g(X, Y) \eta(Z)-g(Y, Z) \eta(X)\}, \\
& S(X, \xi)=-\alpha^{2}(n-1) \eta(X),
\end{aligned}
$$

$$
\begin{aligned}
& S(\xi, \xi)=-\alpha^{2}(n-1), \\
& Q \xi=-\alpha^{2}(n-1) \xi, \\
& \left(\nabla_{X} \eta\right) Y=\alpha\{g(X, Y)-\eta(X) \eta(Y)\},
\end{aligned}
$$

for all vector fields $X, Y, Z$ on $\chi(M)$, where $R$ is the Riemannian curvature tensor, $S$ is the Ricci tensor of type $(0,2)$ and $Q$ is the Ricci operator defined as $S(X, Y)=g(Q X, Y)$.

\section{Parallel symmetric second order tensors and Ricci solitons in $\alpha$-Kenmotsu manifolds}

Let $h$ denote a $(0,2)$ type symmetric tensor field which is parallel with respect to $\nabla$ that is $\nabla h=0$. Then it follows that ${ }^{14,23}$

$$
\nabla^{2} h(X, Y ; Z, W)-\nabla^{2} h(X, Y ; W, Z)=0
$$

which gives

$h(R(X, Y) Z, W)+h(Z, R(X, Y) W)=0$.

Taking $Z=W=\xi$ in (16) and using (8), we have

$\alpha^{2}\{\eta(X) h(Y, \xi)-\eta(y) h(X, \xi)\}=0$.

Since $\alpha$ is non-zero, so by taking $X=\xi$ in (17) and by the symmetry of $h$, we have

$h(Y, \xi)=\eta(Y) h(\xi, \xi)$.

Differentiating (18) covariantly with respect to $X$, we have

$$
\begin{gathered}
\left(\nabla_{X} h\right)(Y, \xi)+h\left(\nabla_{X} Y, \xi\right)+h\left(Y, \nabla_{X} \xi\right) \\
=\left(\nabla_{X} \eta\right)(Y) h(\xi, \xi)+\eta\left(\nabla_{X} Y\right) h(\xi, \xi) \\
+\eta(Y)\left(\nabla_{X} h\right)(\xi, \xi)+2 \eta(Y) h\left(\nabla_{X} \xi, \xi\right) . \\
\text { By using (7), (14), (18) and the parallel } \\
\text { condition } \nabla h=0 \text { in }(19), \text { we have } \\
\quad h(X, Y)=g(X, Y) h(\xi, \xi) .
\end{gathered}
$$

The above equation implies that $h(\xi, \xi)$ is a constant, via (18). So we have the following theorem.

Theorem 1. A symmetric parallel second order covariant tensor in an $\alpha$-Kenmotsu manifold is a constant multiple of the metric tensor.

Corollary 1. A locally Ricci symmetric $(\nabla S=0)$ $\alpha$-Kenmatsu manifold is an Einstein manifold.

Remark 1. The following statements for $\alpha$ Kenmatsu manifold are equivalent

(i) Einstein,

(ii) locally Ricci symmetric,

(iii) Ricci semi-symmetric, that is $R \cdot S=0$.

The implication $(i) \rightarrow(i i) \rightarrow($ iii $)$ is trivial. Now we prove that the implication $($ iii $) \rightarrow(i)$ in more general frame work of $\alpha$-Kenmotsu manifold. Since $R \cdot S=0$, means exactly (16) with $h$ 
replaced by $S$, that is

$$
\begin{gathered}
(R(X, Y) \cdot S)(U, V)=-S(R(X, Y) U, V)- \\
S(U, R(X, Y) V) .
\end{gathered}
$$

Taking $R \cdot S=0$ and putting $X=\xi$ in (21), we have

$S(R(\xi, Y) U, V)+S(U, R(\xi, Y) V)=0$.

In view of (9) and $\alpha \neq 0$, the above equation becomes

$$
\begin{gathered}
\eta(U) S(Y, V)-g(Y, V) S(\xi, V)+\eta(V) S(U, Y)- \\
g(Y, V) S(U, \xi)=0 .
\end{gathered}
$$

Putting $U=\xi$ in (23) and by using (3), (11) and (12), we obtain

$S(Y, V)=-\alpha^{2}(n-1) g(Y, V)$.

This lead the following theorem.

Theorem 2. A Ricci semi-symmetric $\alpha$ Kenmotsu manifold is an Einstein manifold.

Corollary 2. If on an $\alpha$-Kenmotsu manifold the tensor field $\left(\mathcal{L}_{V} g+2 S\right)$ is parallel, then $(g, V, \lambda)$ gives a Ricci soliton.

Proof. A Ricci soliton in $\alpha$-Kenmotsu manifold is defined by (1). Thus $\left(\mathcal{L}_{V} g+2 S\right)$ is parallel. By theorem (1) it is clear that if an $\alpha$ Kenmotsu manifold admits a symmetric paralle] $(0,2)$ tensor, then the tensor is a constant multiple of the metric tensor. Hence $\left(\mathcal{L}_{V} g+2 S\right)$ is a constant multiple of metric tensor $g$ that is $\left(\mathcal{L}_{V} g+2 S\right)(X, Y)=g(X, Y) h(\xi, \xi)$, where $h(\xi, \xi)$ is a non zero constant. It is the application of the theorem (1) to Ricci soliton.

Theorem 3. If a metric $g$ in an $\alpha$-Kenmotsu manifold is a Ricci soliton with $V=\xi$ then it is $\eta$ Einstein.

Proof. Putting $V=\xi$ in (1), we have

$$
\left(\mathcal{L}_{\xi} g\right)(X, Y)+2 S(X, Y)+2 \lambda g(X, Y)=0 \text {, }
$$

where $\left(\mathcal{L}_{\xi} g\right)(X, Y)=g\left(\nabla_{X} \xi, Y\right)+g\left(X, \nabla_{Y} \xi\right)$.

$$
=2 \alpha\{g(X, Y)-\eta(X) \eta(Y)\} \text {. }
$$

Substituting (25) in (24) and by use of (7), we obtain

$S(X, Y)=-(\alpha+\lambda) g(X, Y)+\alpha \eta(X) \eta(Y)$.

Hence the result.

Theorem 4. A Ricci soliton $(g, \xi, \lambda)$ in an $n$ dimentional $\alpha$-Kenmotsu manifold can not be steady but is shrinking.

Proof. In the Linear Algebra either the vector field $V \in \operatorname{Span} \xi$ or $V \perp \xi$. However, the second case seems to be complex to analyse in practice. For this reason, we investigate for the case $V=$ $\xi$. obtain

By a simple computation of $\left(\mathcal{L}_{V} g+2 S\right)$, we

$$
\left(\mathcal{L}_{\xi} g\right)(X, Y)=0
$$

$$
h(\xi, \xi)=-2 \lambda,
$$

where $h(\xi, \xi)=\left(\mathcal{L}_{\xi} g\right)(\xi, \xi)+2 S(\xi, \xi)$.

Using (12) and (26) in above equation, we get

$$
h(\xi, \xi)=2 \alpha^{2}(n-1)
$$

Equating (27) and (29), we have

$$
\lambda=-\alpha^{2}(n-1) \text {. }
$$

Since $\alpha$ is some non-zero scalar function, we have $\lambda \neq 0$, that is Ricci soliton in an $n$ dimensional $\alpha$-Kenmotsu manifold cannot be steady but is shrinking because $\lambda<0$.

Theorem 5. If an $n$-dimensional $\alpha$-Kenmotsu manifold is $\eta$-Einstein then the Ricci solitons in $\alpha$ Kenmotsu manifold that is $(g, \xi, \lambda)$ where $\lambda=$ $-\alpha^{2}(n-1)$ with varying scalar curvature cannot be steady but it is expending.

Proof. The proof consists of three parts.

(i) We prove $\alpha$-Kenmotsu manifold is $\eta$ Einstein,

(ii) We prove the Ricci soliton in $\alpha$-Kenmotsu manifold is consisting of varying scalar curvature,

(iii) We find that the Ricci soliton in $\alpha$ Kenmotsu manifold is expending.

First we prove that the $\alpha$-Kenmotsu manifold is $\eta$-Einstein: the metric $g$ is called $\eta$-Einstein if there exists two real function $a$ and $b$ such that the Ricci tensor of $g$ is given by the general equation

$$
S(X, Y)=a g(X, Y)+b \eta(X) \eta(Y) \text {. }
$$

Let $e_{i},(i=1,2, \ldots n)$ be an orthonormal basis of the tangent space at any point of the manifold. Then putting $X=Y=e_{i}$ in (30) and taking summation over $i$, we get

$$
r=a n+b \text {. }
$$

Again putting $X=Y=\xi$ in (30) then by use of (12), we have

$a+b=-\alpha^{2}(n-1)$.

Then from (31) and (32), we have

$$
a=\left(\alpha^{2}+\frac{r}{n-1}\right), b=-\left(n \alpha^{2}+\frac{r}{n-1}\right) \text {. }
$$

Substituting the value of $a$ and $b$ from (33) in (30), we have

$$
\begin{gathered}
S(X, Y)=\left(\alpha^{2}+\frac{r}{n-1}\right) g(X, Y)-\left(n \alpha^{2}+\right. \\
\left.\frac{r}{n-1}\right) \eta(X) \eta(Y),
\end{gathered}
$$

the above equation shows that $\alpha$-Kenmotsu manifold is $\eta$-Einstein manifold.

Now, we have to show that the scalar curvature $r$ is not a constant and it is varying. 
For an $n$-dimensional $\alpha$-Kenmotsu manifolds the symmetric parallel covariant tensor $h(X, Y)$ of type $(0,2)$ is given by

$h(X, Y)=\left(\mathcal{L}_{\xi} g\right)(X, Y)+2 S(X, Y)$.

By using (25) and (34) in (35), we have

$$
\begin{gathered}
h(X, Y)=2\left\{\alpha(\alpha+1)+\frac{r}{n-1}\right\} g(X, Y)-2\{\alpha(n \alpha+ \\
\left.1)+\frac{r}{n-1}\right\} \eta(X) \eta(Y) .
\end{gathered}
$$

Differentiating (36) covariantly with respect to $Z$ and using (14), we have

$$
\begin{aligned}
\left(\nabla_{Z} h\right)(X, Y)= & 2\left\{(Z \alpha)(\alpha+1)+\alpha(Z \alpha)+\frac{\nabla_{Z} r}{n-1}\right\} g(X, Y) \\
& -2\{(Z \alpha)(n \alpha+1)+n \alpha(Z \alpha)+ \\
& \left.\frac{\nabla_{Z} r}{n-1}\right\} \eta(X) \eta(Y) \\
- & 2\left\{\alpha(n \alpha+1)+\frac{r}{n-1}\right\} \alpha\{g(Z, X)- \\
& \eta(Z) \eta(X) \\
& +g(Z, Y)-\eta(Z) \eta(Y)\} .
\end{aligned}
$$

By substituting $Z=\xi$ and $X=Y \in(\text { Span })^{\perp}$ in

(37) and by using $\nabla h=0$, we have

$\nabla_{\xi} r=-(n-1) \nabla_{\xi}\{\alpha(\alpha+1)\}$.

On integrating (38), we have

$r=-(n-1) \alpha(\alpha+1)+c$,

where $c$ is some integral constant. Thus from (39), we have $r$ is a varying scalar curvature.

Finally, we have to check the nature of the soliton that is Ricci soliton in $\alpha$-Kenmotsu manifold:

From (1), we have $h(X, Y)-2 \lambda g(X, Y)$ then putting $X=Y=\xi$, we have

$h(\xi, \xi)=-2 \lambda$.

On putting $X=Y=\xi$ in (36), we have

$h(\xi, \xi)=-2(n-1) \alpha^{2}$.

Equating (40) and (41), we have

$\lambda=(n-1) \alpha^{2}$.

This show that $\lambda>0, \forall n>1$ and hence Ricci soliton in an $\alpha$-Kenmotsu manifold is expending.

Theorem 6. If a Ricci soliton $(g, \xi, \lambda)$ where $\lambda=$ $2 \alpha^{2}$ of 3 -dimensional $\alpha$-Kenmotsu manifold with varying scalar curvature cannot be steady but it is expending.

Proof. The proof consists of three parts.

(i) We prove that the Riemannian curvature tensor of 3-dimensional $\alpha$-Kenmotsu manifold is $\eta$-Einstein,

(ii) We prove that the Ricci soliton in 3dimensional $\alpha$-Kenmotsu manifold is consisting of varying scalar curvature,

(iii) We prove that find that the Ricci soliton in a 3-dimentional $\alpha$-Kenmotsu manifold is expending.

The Riemannian curvature tensor of 3- dimensional $\alpha$-Kenmotsu manifold is given by $R(X, Y) Z=g(Y, Z) Q X-g(X, Z) Q Y+S(Y, Z) X-$

$$
S(X, Z) Y \quad-\frac{r}{2}\{g(Y, Z) X-g(X, Z) Y\} .
$$

Putting $Z=\xi$ in (42) and by using (8) and (11), we have

$$
\begin{gathered}
\alpha^{2}\{\eta(X) Y-\eta(Y) X\}=\eta(Y) Q X-\eta(X) Q Y- \\
\left(2 \alpha^{2}+\frac{r}{2}\right)\{\eta(Y) X-\eta(X) Y\} .
\end{gathered}
$$

Again putting $Y=\xi$ in (43) and by using (2), (3) and (13), we get

$$
Q X=\left(\alpha^{2}+\frac{r}{2}\right) X-\left(3 \alpha^{2}+\frac{r}{2}\right) \eta(X) \xi .
$$
have

By taking an inner product with $Y$ in (44), we

$$
S(X, Y)=\left(\alpha^{2}+\frac{r}{2}\right) g(X, Y)-\left(3 \alpha^{2}+\frac{r}{2}\right) \eta(X) \eta(Y) .
$$

It shows that 3-dimensional $\alpha$-Kenmotsu manifold is $\eta$-Einstein manifold.

Now, we have to show that the scalar curvature $r$ is not a constant that is $r$ is varying

We have

$h(X, Y)=\left(\mathcal{L}_{\xi} g\right)(X, Y)+2 S(X, Y)$.

By using (25) and (45) in (46), we have

$$
\begin{gathered}
h(X, Y)=2\left\{\alpha(\alpha+1)+\frac{r}{2}\right\} g(X, Y)-2\{\alpha(3 \alpha+ \\
\left.1)+\frac{r}{2}\right\} \eta(X) \eta(Y) .
\end{gathered}
$$

Differentiating above equation with respect to $Z$, we have

$$
\begin{aligned}
\left(\nabla_{Z} h\right)(X, Y)= & 2\left\{(Z \alpha)(\alpha+1)+\alpha(Z \alpha)+\frac{\nabla_{Z} r}{2}\right\} g(X, Y) \\
- & 2\{(Z \alpha)(3 \alpha+1)+\alpha(3 Z \alpha)+ \\
& \left.\frac{r}{2}\right\} \eta(X) \eta(Y) \\
- & 2\left\{\alpha(3 \alpha+1)+\frac{r}{2}\right\}\left\{\left(\nabla_{Z} \eta\right)(X) \eta(Y)+\right. \\
& \left.\eta(X)\left(\nabla_{Z} \eta\right)(Y)\right\} .
\end{aligned}
$$

By substituting $Z=\xi$ and $X=Y \in(\text { Span })^{\perp}$ in (48) and by using $\nabla h=0$, we have

$\nabla_{\xi} r=-2 \nabla_{\xi}\{\alpha(\alpha+1)\}$.

On integrating (49), we have

$r=-2 \alpha(\alpha+1)+c$,

where $c$ is some integral constant. Thus from (50), we have $r$ is a varying scalar curvature.

Finally we have to check the nature of the Ricci soliton $(g, \xi, \lambda)$ in 3-dimensional $\alpha$ Kenmotsu manifold.

From (1), we have $h(X, Y)-2 \lambda g(X, Y)$ then putting $X=Y=\xi$, we have

$h(\xi, \xi)=-2 \lambda$.

On putting $X=Y=\xi$ in (47), we have

$h(\xi, \xi)=-4 \alpha^{2}$.

Equating (51) and (52), we have 
Equating (51) and (52), we have $\lambda=2 \alpha^{2}$.

This show that $\lambda>0$ and hence Ricci soliton in an $\alpha$-Kenmotsu manifold is expending.

\section{References}

I. Hamilton, R.S. (1988). The Ricci flow on surfaces. Math and general relativity (Santa Cruz, CA, 1986), Contemporary Mathematics. 71, 237-262.

2. Chave, T. \& Valent, G. (1996). Quasi-Einstein metrics and their renormalizability properties. Helvetica Physica Acta. 69, 344-347.

3. Chave, T. \& Valent, G. (1996). On a class of compact and non-compact quasi-Einstein metrics and their renormalizability properties. Nuclear Physics. B 478, 758778 .

4. Chow, B., Lu, P. \& Ni, L. (2006). Hamilton's Ricci flow. Graduate Studies in Mathematics, American Mathematical Society, Providence, RI, USA, 77.

5. Das, L. (2007). Second order parallel tensors onSasakian manifold, Acta Mathematica Academiae Paedagogicae Nyíregybáziensis, 23, 65-69.

6. Sharma, R. (2008). Certain results on K-contact and $(\kappa, \mu)$-contact manifolds. Journal of Geometry, 89, 138 I47.

7. Ghosh, A., Sharma, R. \& Cho, J. T. (2008). Contact metric manifolds with $\eta$-parallel torsion tensor. Annals of Global Analysis and Geometry, 34, 287-299.

8. De, U. C. (2010). Ricci soliton and gradient Ricci soliton on P-Sasakian manifolds. The Aligarh Bulletin of Mathematicss. 29, 29-34.

9. Barua, B. \& De. U. C. (2013). Characterizations of a Riemannian manifold admitting Ricci solitons. Facta Universitatis (NIS) Series: Mathematics and Informatics, 28, 127-I32.

Io. Calin, M. \& Crasmareanu, M. (2010). From the Eisenhart Problem to Ricci solitons in $f$-Kenmotsu mani- folds. Bulletin of the Malaysian Mathematical Sciences Society. 33, 36I-368.

II. Blaga, A. M. (2015). Eta-Ricci soliton on paraKenmotsu manifold, Balkan Journal of Geometry and its Applications. 20, I-3I.

I2. Bagewadi, C. S. \& Ingalahalli, G. (20I2). Ricci solitons in Lorentzian $\alpha$-Sasakian manifolds. Acta Mathematica Academiae Paedagogicae Nyíregybáziensis 28, 59-68.

13. Sharma, R. (1989). Second order parallel tensor in real and complex space forms. International Journal of Mathematics and Mathematical Sciences, 12, 787-790.

I4. Futaki, A., Ono, H. \& Wang, G. (2009). Transverse Kähler geometry of Sasaki manifolds and toric SasakiEinstein manifolds. Journal of Differential Geometry, 83, 585-636.

I5. He, C. \& Zhu, M. (20II). The Ricci solitons on Sasakian manifolds, arxiv:1109.4407v2.

16. Ingalahalli, G. \& Bagewadi, C. S. (2012). Ricci solitons in $\alpha$-Sasakian manifolds, International Scholarly Research Notices: Geometry. Article ID 421384, I3-pages.

17. Kim, T. W. \& Pak, H. K. (2005). Canonical foliations of certain class of almost contact metric structures. Acta Mathematica Sinica, English Series, 21, 84I-846.

I8. Janssens, D. \& Vanhecke, L. (198I). Almost contact structures and curvature tensors. Kodai Mathematical Journal, 4, I-27.

19. Blair, D.E. (2002). Riemannian geometry of contact and symplectic manifolds. Progress in Mathematics, Birkbâuser, 203.

2o. Öztürk, H., Aktam, N. \& Murathan, C. (20I0). On $\alpha$ Kenmotsu manifolds satisfying certain conditions. Applied Sciences, I2, II5-I26.

2I. Kumar, R. (2015). Some results on $\alpha$-Kenmotsu manifold. Science and Technology Journal, 3, 179-182.

22. Topping, P. (2006). Lectures on the Ricci Flow. In: London Mathematical Society Lecture Note Series. Cambridge University Press, Cambridge, UK, p. 325. 